\title{
TOMOGRAFIA COMPUTADA DE ALTA RESOLUCION EN NIÑOS CON DAÑO PULMONAR POR ADENOVIRUS: CARACTERIZACION Y CORRELACION CON FUNCION PULMONAR*
}

\section{Drs. Gloria Soto G, Marcela Linares P, Juan C. Díaz P, Juan A. Escaffi J, Fernando Pedersen M, Noldy Mardones} C, D. Busel M, Samuel Gasc H, T.M. Pablo Arancibia N.

Hospital Padre Hurtado, Santiago, Chile

*Trabajo premiado por el comité de selección en el Congreso de Radiología 2002.

\begin{abstract}
Introduction: Respiratory tract infections caused by Adenovirus (ADV) can lead to long term lung sequelae. In our media it is the most frequent cause of chronic lung disease in children. High resolution computed tomography (HRCT) can characterize the anatomic alterations of this disease. Functional impairment is evaluated with spirometry, which only can be performed in collaborating patients. Purpose: Characterize the appearances of chronic lung disease secondary to ADV infection on HRCT, relate the extent of HRCT abnormalities to pulmonary function impairment, and determine if HRCT can be used to assess pulmonary function when functional tests cannot be performed. Material and methods: HRCT findings in 17 pediatric patients with post adenoviral chronic lung disease were analyzed. HRCT findings were correlated with spirometry in 11 of these children using the modified Bhalla score for bronchial dilatation and the modified Siegel score for mosaic lung attenuation. Results: HRCT findings were mosaic perfusion and bronchial wall thickening in 17/17, bronchial dilatation and centrilobular nodules in 16/ 17 , atelectasis in 11/17, and tree in bud in 8/17. Very good correlation was found between the scores of mosaic lung attenuation and bronchial dilatation with the forced expiratory volume in one second. Conclusion: A characteristic pattern of findings on $H R C T$ is present in children with post adenovirus chronic lung disease. There is good correlation between the severity of mosaic lung attenuation and bronchial dilatation with pulmonary functional
\end{abstract}

Soto G. y cols. Tomografía computada de alta resolución en niños con daño pulmonar por adenovirus: Caracterización y correlación con función pulmonar. Rev Chil Radiol 2002; 8: 149-153.

Correspondencia: Dra. Gloria Soto G.

gsoto@alemana.cl impairment. These results suggest that HRCT findings can be used to assess pulmonary function in those children in which pulmonary function tests cannot be performed.

Key words: Adenovirus, Bronchiolitis obliterans, Pulmonary function.

\section{Introducción}

La infección respiratoria por adenovirus (ADV) puede producir daño pulmonar persistente y progresivo. En nuestro medio es la causa más frecuente de daño pulmonar crónico en niños. Clínicamente se caracteriza por el desarrollo de un cuadro de obstrucción de la vía aérea persistente y progresivo que no responde a broncodilatadores ni a la inhalación de corticoides. Histológicamente la lesión corresponde a una bronquiolitis constrictiva, caracterizada por grados variables y progresivos de inflamación de la vía aérea periférica con obliteración cicatricial de su lumen ${ }^{(1)}$. Funcionalmente determina compromiso obstructivo de la vía aérea el que puede ser cuantificado mediante espirometría.

En la literatura hay escasas referencias respecto al rol y el rendimiento de la tomografía computada de alta resolución (TCAR) en niños con secuelas de $A D V^{(2,3)}$, y de acuerdo a nuestro conocimiento, no existen estudios que evalúen la asociación entre las alteraciones encontradas y el compromiso de la función pulmonar evaluada mediante espirometría.

\section{Objetivos}

Los objetivos de este trabajo son: caracterizar los hallazgos en TCAR en niños con daño pulmonar secundario a ADV, correlacionar la severidad de las alteraciones con el compromiso de la función pulmonar y determinar si la TCAR permite inferir el compromiso funcional en aquellos niños en los que no es posible estudiar directamente la función pulmonar. 


\section{Materiales y método}

Se evaluaron retrospectivamente las fichas clínicas, TCAR y pruebas funcionales de todos los niños con diagnóstico clínico de daño pulmonar secundario a ADV estudiados en nuestro servicio.

El diagnóstico clínico se fundamentó en un cuadro permanente y progresivo de obstrucción de la vía aérea, en niños con antecedentes de una neumonía grave por ADV en los que se descartó otras causas de patología obstructiva crónica, como fibrosis quística o inmunodeficiencias.

Pacientes: El universo corresponde a 17 niños, 11 varones y 6 mujeres, con un rango de edad de 1 a 15 años (promedio de 7 años 7 meses). En todos ellos el cuadro inicial de infección por ADV ocurrió en el transcurso de los primeros dos años de vida. El tiempo de evolución de la patología esta entre los 9 meses y 14 años.

Tomografía computada: Las TCAR fueron realizadas con los pacientes en condiciones clínicas estables, sin cuadros pulmonares intercurrentes. Se usó un Tomógrafo Philips Tomoscan AV Performance. Se utilizó un protocolo de alta resolución: tiempo de adquisición de 1 segundo, imágenes de $1 \mathrm{~mm}$ de grosor procesadas con algoritmo de alta resolución espacial (Bone). Los intervalos de corte fueron de 10 a 20 $\mathrm{mm}$ dependiendo de la edad del paciente. En $14 \mathrm{ca}$ sos se obtuvo imágenes en inspiración y espiración; en los restantes casos solo en inspiración. En 4 niños, menores de 4 años, los exámenes se realizaron con sedación anestésica. En el resto de los pacientes no se utilizo sedación.

Las TCAR fueron analizadas en forma independiente por 2 radiólogos (G.S., J.C.D) sin tener conocimiento de los resultados de las pruebas funcionales. El informe final se hizo por consenso.

Función pulmonar: La función pulmonar se evaluó mediante espirometría en 11 niños. En 6 niños no fue posible efectuar la espirometría por falta de colaboración ( 4 niños menores de 4 años y 2 niños, de 6 y 8 años, portadores de síndrome de Down). Las espirometrías se realizaron cumpliendo los criterios de aceptabilidad y reproducibilidad de la Sociedad Americana de Tórax. En 7 niños el examen se efectuó el mismo día que la TC, en 3 el lapso entre ambos exámenes fue de entre 7 y 30 días, y en un caso de 54 días.

\section{Correlación TCAR/ Función pulmonar}

Para correlacionar las alteraciones de la TCAR con el compromiso funcional se cuantifico la extensión y severidad de la dilatación bronquial, y la extensión del parénquima pulmonar comprometido con mosaico de perfusión. La dilatación bronquial se cuantificó en base al score de Bhalla modificado(1) y la extensión de mosaico utilizando el score de Siegel modificado(2).
Para la obtención de estos scores cada pulmón se divide en 3 áreas (lóbulo superior, lóbulo medio y lóbulo inferior en el pulmón derecho. Lóbulo superior sin língula, língula y lóbulo inferior en el pulmón izquierdo).

Para el score de dilatación bronquial a cada área se le asigna 0 puntos si no hay bronquios dilatados, 1 punto si hay bronquios dilatados de menos de 5 $\mathrm{mm}$ de diámetro, 2 puntos si los bronquios dilatados tienen diámetro de entre 5 y $10 \mathrm{~mm}$ de diámetro, y 3 puntos si el diámetro bronquial es de más de $10 \mathrm{~mm}$.

Para el score de mosaico se asigna 0 puntos en ausencia de mosaico, 1 punto si el parénquima de menor atenuación compromete menos de $33 \%$ del área, 2 puntos si compromete entre 33 y $66 \%$ del parénquima, y 3 puntos si el compromiso es de más de $66 \%$ del área. Los lóbulos completamente atelectásicos no fueron considerados en la determinación del score de mosaico. El score final para cada alteración corresponde a la suma del puntaje de todas las áreas dividido por el número de áreas evaluadas, con un rango teórico de 0 a 3 .

El parámetro de la espirometría utilizado en la correlación fue el VEF ${ }_{1}$ Para el análisis estadístico se utilizó el coeficiente correlación de Spearman. Coeficientes de 0 a 0.25 indican correlación escasa o ausencia de correlación; de 0.25 a 0.5 indican cierto grado de correlación; de 0.5 a 0.75 la correlación es de moderada a buena, y mayor de 0.75 es muy buena a excelente.

\section{Resultados}

Hallazgos TCHR: Se observó mosaico de perfusión y engrosamiento de paredes bronquiales en todos los pacientes, dilatación bronquial y nódulos centrolobulillares en 16, atelectasias en 11, y opacidades tipo "árbol en brote" en 8.

El patrón de mosaico de perfusión del parénquima pulmonar (Figura 1) fue un hallazgo constante y siempre bilateral. En 15 niños el mosaico comprometía tres o más lóbulos pulmonares siendo en 10 de ellos el compromiso de todos los lóbulos. En la totalidad de los 14 casos en que se dispuso de imágenes obtenidas en espiración se observó atrapamiento aéreo en las áreas de menor atenuación (Figura 2). En 5 niños, en un total de 9 lóbulos, el atrapamiento aéreo observado en espiración hizo evidente mosaico no detectado en las imágenes en inspiración.

Se detectó dilatación bronquial en 16 de los 17 niños, bilateral en 13 casos. En 12 casos los bronquios dilatados tenían diámetro que no superaban los $5 \mathrm{mms}$. En los restantes 4 casos se observó bronquios de más de $10 \mathrm{~mm}$ de diámetro.

En todos los pacientes había engrosamiento de paredes bronquiales, tanto en relación a bronquios dilatados como a bronquios de calibre normal (Figura 3). 
En 16 pacientes se encontraron nódulos centrolobulillares y en 8 imágenes de "árbol en brote" (Figura 4). Estas opacidades centrolobulillares fueron siempre poco profusas.

Once de los 17 pacientes presentaban atelectasias. En 9 se observo atelectasias segmentarias o subsegmentarias las que fueron de
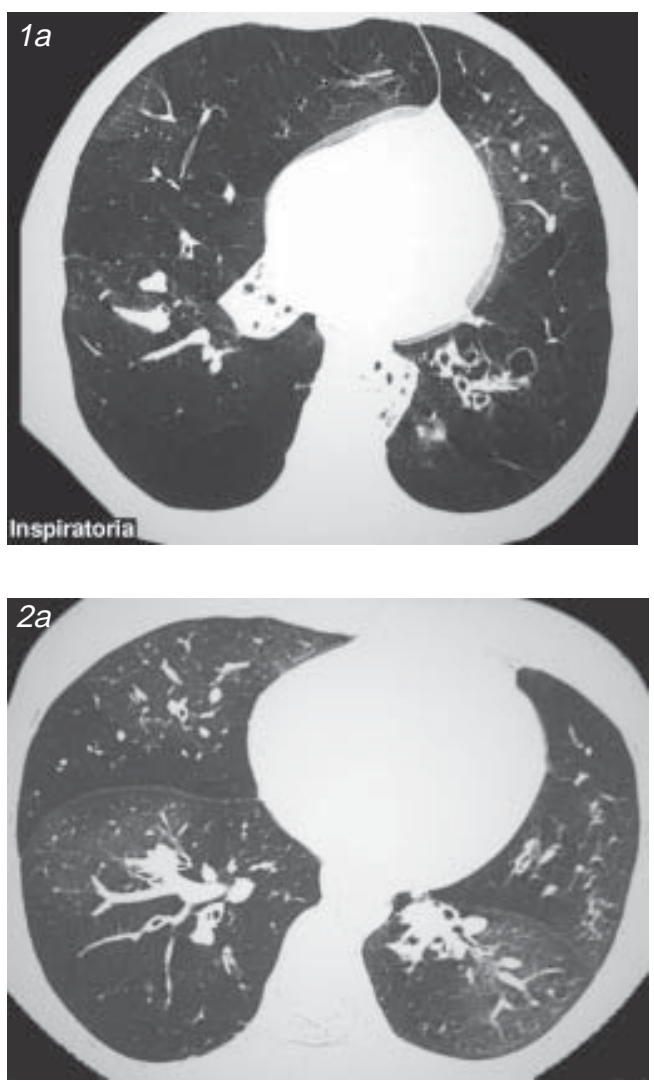

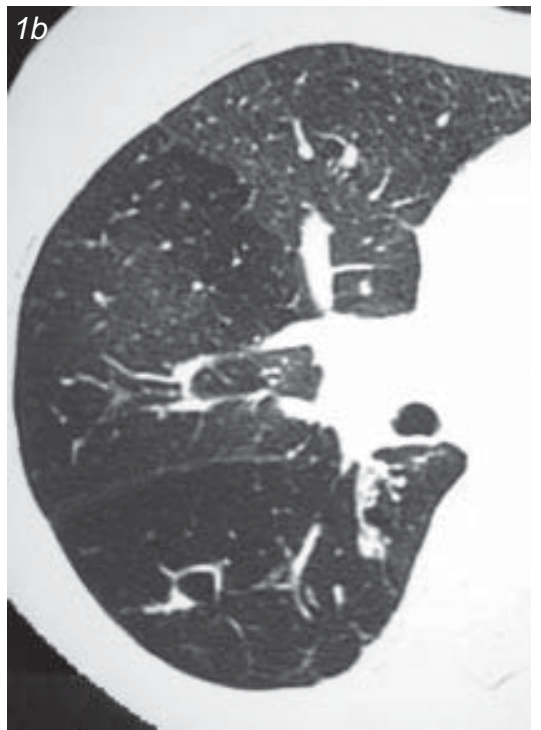

Figura 1 a,b. Cortes de alta resolución que muestran zonas parchadas de mayor densidad respetando los límites lobulillares. Las zonas de menor densidad muestran menor número y calibre de las estructuras vasculares, hallazgo característico del «mosaico de perfusión».

Figura 2 a,b. Cortes de alta resolución al mismo nivel en inspiración (a) y espiración (b). En espiración el patrón en mosaico se hace más evidente por atrapamiento de aire en las zonas comprometidas. Este es un recurso que ayuda en la diferenciación entre patología vascular y de la vía aérea.

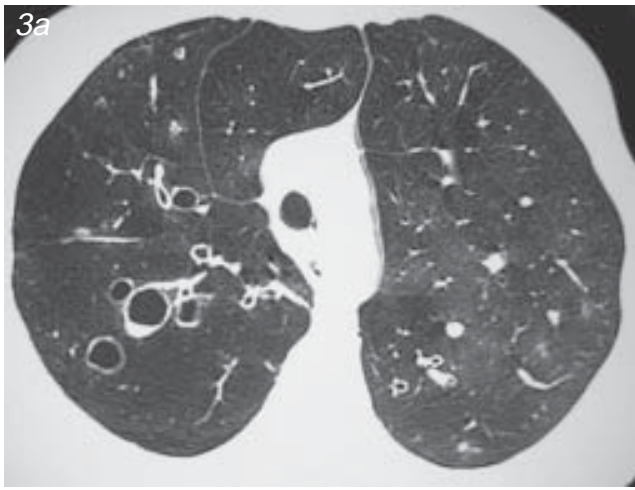

Figura 3. Engrosamiento de paredes tanto de bronquios dilatados, de mayor calibre que los vasos acompañantes, como de bronquios de calibre normal.

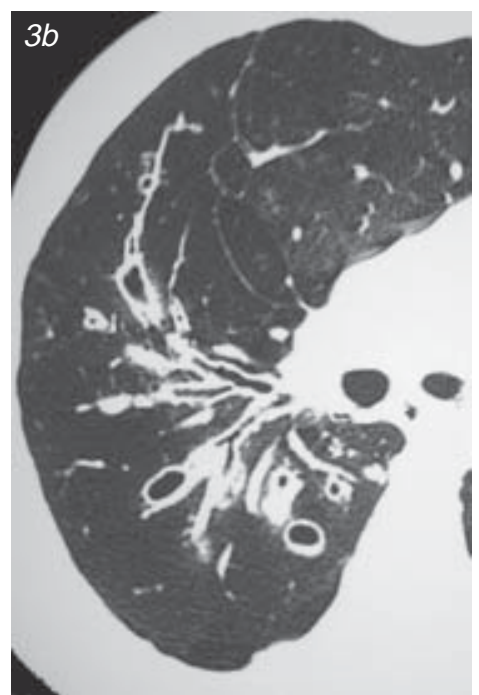

Figura 4. Nódulos centrolobulillares y opacidades en «árbol en brote». Estas últimas corresponden bronquiolos dilatados, con impactación mucosa. 
distribución aleatoria (Figura 5a). Tres niños tenían atelectasias lobares, en un caso ambos lóbulos inferiores, en los otros dos del lóbulo inferior izquierdo (Figura 5b). Todos los lóbulos atelectásicos presentaban dilatación bronquial severa.
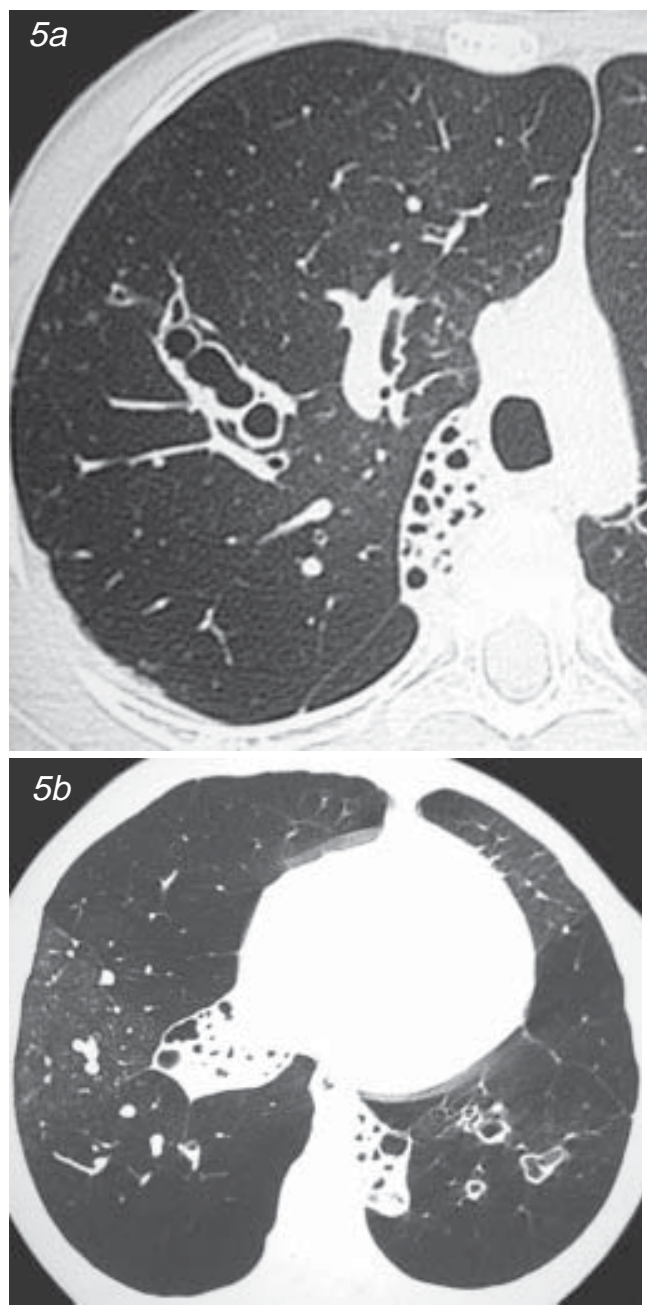

Figura 5a,b. Atelectasia segmentaria del lóbulo superior derecho (a), y de ambos lóbulos inferiores (b). Se observa dilatación importante de bronquios en los lóbulos atelectásicos.

Espirometría: Todos los pacientes presentaron compromiso funcional obstructivo de la vía aérea el que fue severo en 6 casos $\left(\mathrm{VEF}_{1}<50 \%\right)$, moderado en 1 caso $\left(\mathrm{VEF}_{1} 50-65 \%\right)$, y leve en $4\left(\mathrm{VEF}_{1}>65 \%\right)$.

Correlación TCAR / Función pulmonar. El coeficiente de correlación de Spearman entre el score de dilatación bronquial y el VEF ${ }_{1}$ fue de de 0.80 , considerado como una muy buena correlación entre ambos parámetros (Grafico 1).

El coeficiente de correlación de Spearman entre el score de mosaico y el VEF fue de 0.86 , considerado como una correlación excelente entre ambos parámetros (Grafico 2). Un score de mosaico de 1.83 discriminó entre niños con obstrucción severa de aquellos con obstrucción leve o moderada (Grafico 3).

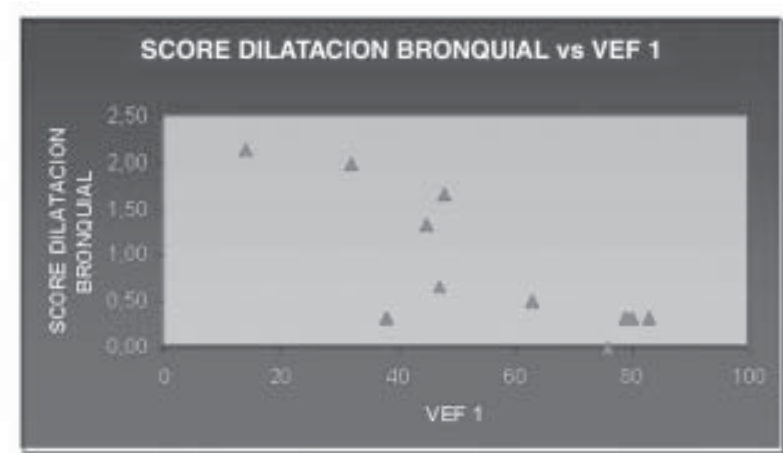

Gráfico 1. Correlación score dilatación bronquial vs VEF

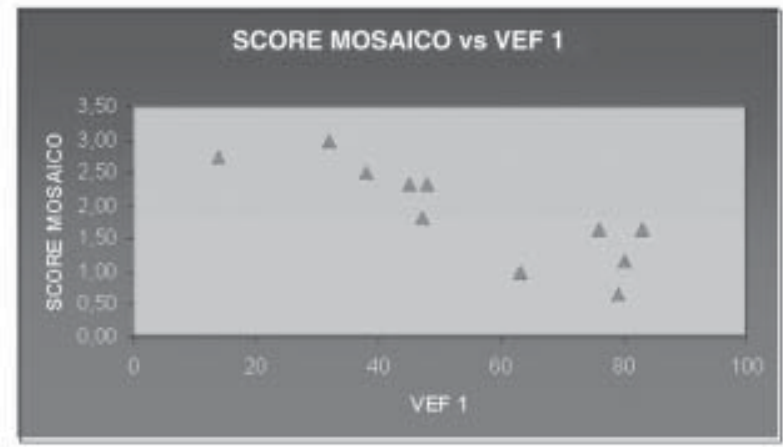

Gráfico 2. Correlación score mosaico vs VEF

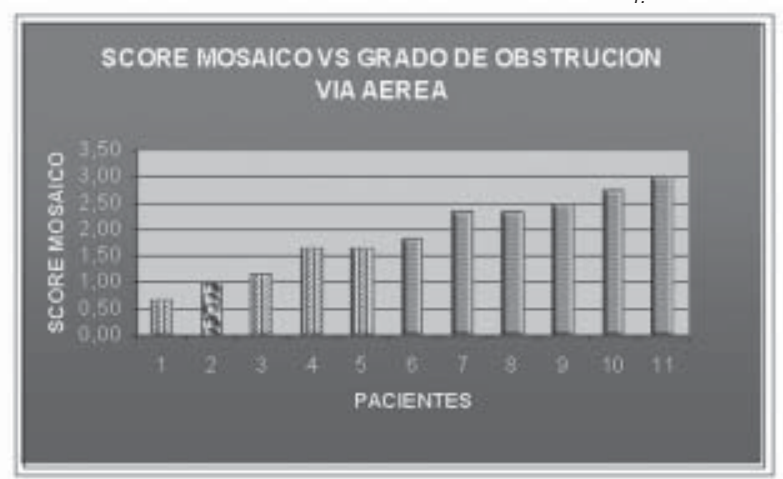

Gráfico 3. Score mosaico vs severidad de obstrucción bronquial. Un score de mosaico de 1,833 discrimina los pacientes con obstrucción severa (ए) de aquellos con obstrucción moderada (\$) o leve (非)

\section{Discusión}

La infección respiratoria por ADV es, en nuestro medio, la causa mas frecuente de daño pulmonar crónico en niños. Histológicamente la alteración corresponde a una bronquiolitis constrictiva, caracterizada por grados variables y progresivos de inflamación de la vía aérea pequeña con obliteración cicatricial de su lumen ${ }^{(3)}$. Por ser la vía aérea pequeña no visible radiológicamente la radiografía de tórax puede ser normal, o mostrar solo alteraciones inespecíficas tales como hiperinsuflación, engrosamiento peribronquial y bronquiectasias.

Lynch y cols. demostraron la superioridad de la TCAR sobre la radiografía de tórax en la detección de alteraciones pulmonares en pacientes adultos con bronquiolitis constrictiva post infecciosa ${ }^{(4)}$. Actualmente la TCAR es el método de elección en el estudio de pacientes adultos con 
bronquiolitis constrictiva. En la literatura hay pocos referencias respecto al rol y el rendimiento de la TCAR en niños con secuelas de $\operatorname{ADV}(4,5)$.

Nuestra serie muestra un patrón característico de alteraciones en TCAR en niños con daño pulmonar crónico secundario a infección por ADV. Las alteraciones más constantes, presentes en más del $94 \%$ de los casos, son el mosaico de perfusión, la dilatación bronquial, el engrosamiento de la paredes bronquiales y la presencia de nódulos centrolobulillares. Hallazgos menos constantes son la presencia de atelectasias, más frecuentemente segmentarias y subsegmentarias, y las opacidades tipo "árbol en brote", obsevados en cerca del $50 \%$ de los casos en esta serie.

El engrosamiento de las paredes bronquiales, presente en todos los casos, puede estar determinado por engrosamiento de la pared bronquial propiamente $\mathrm{y} / \mathrm{o}$ del intersticio peribronquia|(5).

Otro hallazgo constante en nuestra serie fue el mosaico de perfusión, caracterizado por áreas de mayor y menor atenuación del parénquima pulmonar, con menor profusión y calibre de estructuras vasculares en las áreas de menor atenuación. En todos los exámenes en que se obtuvo imágenes en espiración se demostró atrapamiento aéreo en las áreas de menor atenuación, hallazgo descrito en la literatura(6,7). En 5 de nuestros pacientes observamos que las imágenes obtenidas en espiración son mas sensibles en la detección de mosaico ${ }^{(8,9)}$, por lo que a nuestro juicio el protocolo de estudio de estos pacientes debiera siempre incluirlas.

La dilatación bronquial fue hallazgo muy frecuente, presente en 16 de los 17 casos. Al igual que en otras publicaciones $^{(8)}$ las bronquiectasias fueron en general leves y con mayor frecuencia de distribución bilateral. Si bien en nuestro trabajo no relacionamos la severidad de las mismas con las características del cuadro clínico, Zhang et al ${ }^{(8)}$ demostraron correlación entre la magnitud y extensión de la dilatación bronquial con la severidad del cuadro inicial de la infección por ADV, y no encontraron relación entre la severidad de las bronquiectasias y el tiempo de evolución de la patología.

Las atelectasias, presentes en $64 \%$ de los casos, fueron con mayor frecuencia segmentarias y subsegmentarias; en 4 casos (17.6\%), observamos atelectasias lobares. Estos hallazgos permiten plantear que en esta patología, con frecuencia existe, además del compromiso característico de la vía aérea pequeña, compromiso inflamatorio en bronquios de mediano y gran calibre.

Los nódulos centrolobulillares, presentes en 16 de los 17 casos, pueden estar determinados por una alteración del bronquiolo respiratorio o del intersticio peribronquiolar. Las opacidades tipo "árbol en brote" fueron menos frecuentes en nuestra serie. Corresponden a un signo directo de bronquiolitis y están determinadas por bronquiolos dilatados y con impactación mucosa.

En los 11 casos en que correlacionamos las alteraciones de la TCAR con la función pulmonar, observamos muy buena correlación entre la severidad del compromiso obstructivo de la vía aérea y la extensión y severidad de la dilatación bronquial, de acuerdo al Score de Bhalla modificado. Observamos además que la presencia de bronquios moderada o severamente dilatados, de más de $10 \mathrm{~mm}$ de diámetro, se asoció siempre a compromiso funcional severo de la función pulmonar. La correlación entre el compromiso funcional y la extensión del mosaico de perfusión fue aún mejor. Utilizando el Score de Siegel modificado, en nuestra serie, un valor de 1.833 permitió discriminar entre los pacientes con compromiso severo de la función pulmonar respecto a aquellos con compromiso leve o moderado. Esta observación debe ser validada con estudios con mayor número de pacientes.

\section{Conclusión}

En niños con daño pulmonar secundario a infección por ADV la TCAR muestra un patrón característico de alteraciones caracterizado por mosaico de perfusión, dilatación bronquial, engrosamiento de paredes bronquiales y opacidades centrolobulillares. En forma menos constante se observan atelectasias predominantemente segmentarias y subseg-mentarias. Tanto la extensión del mosaico de perfusión como la extensión y severidad de la dilatación bronquial son parámetros que se correlacionan muy bien con el compromiso de la función pulmonar en estos niños. De acuerdo a estos resultados pensamos que la TCAR puede ser útil para inferir el compromiso de función pulmonar en aquellos niños en los que no es posible realizar pruebas funcionales, situación no infrecuente que ocurrió en 6 de los 17 pacientes de nuestra serie.

\section{Agradecimientos}

Los autores agradecen al Sr. Jorge Rodríguez por su asesoría en el análisis estadístico.

\section{Bibliografía}

1. Bhalla M, Turcios N, Aponte V, Jenkins M. et al. Cystic fibrosis: Scoring system with thin section CT. Radiology 1991; 179: 783-8.

2. Siegel M, Bhalla S, Gutierrez F. et al. Post-lung transplantation bronchiolitis obliterans syndrome: Usefulness of expiratory thin section CT for diagnosis. Radiology 2001;220: 455-462.

3. Mauad T, Dolhnikoff M. Histology of childhood bronchiolitis obliterans. Pediatr Pulmonol 2002; 33: 466-74.

4. Lynch DA, Brasch RC, Hardy KA, Webb WR. Pediatric pulmonary disease: Assessment with high resolution ultrafast CT. Radiology 1990; 176: 243-8.

5. Zhang L, Irion K, da Silva Porto N, Abreu e Silva F. HRCT in pediatric patients with post infectious bronchiolitis obliterans. J Thoracic Imaging 1999; 14: 85-89.

6. Chang A, Masel J, Masters B. Post-infectious bronchiolitis obliterans: Clinical, radiological and pulmonary function sequelae. Pediatr Radiol 1998; 28: 23-29.

7. Lau DM, Siegel MJ, Hildebolt CF, Cohen AYH. Bronchiolitis obliterans syndrome: Thin section CT diagnosis of obstructive changes in infants and young children after lung transplantation. Radiology 1998; 208: 783-788.

8. Lucaya J, Garcia-Peña P, Herrera L. et al. Expiratory chest CT in children. AJR 2000; 174: 235-241. 